\title{
FLUXOS DE EMERGÊNCIA DE AZEVÉM EM SISTEMA DE PRODUÇÃO INTEGRAÇÃO LAVOURA-PECUÁRIA
}

\section{FLOW EMERGENCE OF RYEGRASS IN CROP-LIVESTOCK INTEGRATION SYSTEM}

\author{
Vitor SPADER ${ }^{1}$ \\ Edina Cristiane Pereira LOPES ${ }^{2}$ \\ Eliseu Geraldo dos Santos FABBRIN ${ }^{2}$ \\ Élide Dalzzotto COSTA ${ }^{2}$ \\ Cristina Gonçalves de MENDONÇA ${ }^{3}$ \\ Adelino PELISSARI ${ }^{3}$
}

\begin{abstract}
RESUMO
O objetivo deste trabalho foi determinar os fluxos de emergência do azevém, em área de integração lavoura pecuária. O estudo foi desenvolvido no município de Guarapuava-PR, latitude $25^{\circ} 23^{\prime} 36^{\prime \prime}$ S, longitude $51^{\circ} 27^{\prime} 19^{\prime \prime}$ W, altitude de $1100 \mathrm{~m}$. O solo do local é Latossolo Bruno Distroférrico e o clima Cfb. O delineamento experimental foi o de blocos ao acaso, com seis repetições. Os tratamentos foram constituídos por 9 épocas de quantificação da emergência do azevém, as quais foram realizadas com a contagem das plântulas emergidas na área de um quadrado de ferro com $0,5 \mathrm{~m}^{2}(0,71 \times 0,71 \mathrm{~m})$ aleatoriamente dentro de cada unidade experimental. Após cada avaliação, em toda a área experimental, foi aplicado o herbicida paraquat $200 \mathrm{~g}$ i.a. (1,1'-dimetil-4,4'-bipiridilio dicloreto) (Gramoxone 200- Syngenta Proteção de Cultivos Ltda.), na dosagem de até $0,5 \mathrm{~L} \mathrm{ha}^{-1}$. Atribuiu-se o valor de 100 ao somatório total de indivíduos observados durante todo o período de avaliação e calculou-se o percentual observado em cada época de avaliação em relação a este valor. Na data da primeira avaliação, verificou-se um maior fluxo de emergência, apresentando cerca de $65 \%$ de plântulas, sendo que a partir da aplicação do herbicida paraquat observou-se uma severa diminuição de plântulas emergidas na área ao longo do tempo. Os resultados demonstraram que os meses de outono e inverno apresentaram os menores valores de precipitação, sendo que o mês de agosto apresentou índice acima da média mensal anual, entretanto não apresentou reflexos diretos na emergência das plântulas de azevém.
\end{abstract}

Palavras-chave: Banco de sementes, cereais de inverno, paraquat

\begin{abstract}
The objective of this study was to determine the flow of emergence of ryegrass in crop livestock integration area. The work was conducted in Guarapuava-PR, lat $25^{\circ} 23^{\prime} 36^{\prime \prime}$, long $51^{\circ} 27^{\prime} 19^{\prime \prime}$, alt $1100 \mathrm{~m}$. The soil is Latosols Distroferric and $\mathrm{Cfb}$ climate. The experimental design was randomized block with six replicates, the treatments were 11 times of flow emergency quantification, were performed by counting emerged plants in the area of a square of iron with $0,5 \mathrm{~m}^{2}$, randomly within each experimental unit. After each evaluation, throughout the experimental area was applied paraquat to $200 \mathrm{~g}$ a.i. (1,1'-dimetil-4,4'-bipiridilio dicloreto) (Gramoxone 200Syngenta Proteção de Cultivos Ltda.) dose of up to $0,5 \mathrm{~L} \mathrm{ha}{ }^{-1}$. We gave it the value 100 to total sum of individuals observed at each time of assessment in relation to this value. In the first evaluation date, there is a flow emergency about $65 \%$ of seedlings, and from application of paraquat was observed a decrease in the seedling emerged in the area over the time. The results showed that the months of autumn and winter had the lowest values of rainfall, and the month of august has a rainfall index above the average monthly annual, however showed no direct effects on ryegrass emergence.
\end{abstract}

Key-words: Seed bank, winter cereals, paraquat

\footnotetext{
Engo-Agr'. Doutorando do Programa de Pós-graduação em Produção Vegetal da UFPR. Pesquisador da Fundação Agrária de Pesquisa Agropecuária - FAPA. Guarapuava, PR, Brasil. Email: vspader@agraria.com.br

Eng ${ }^{\circ}$-Agr ${ }^{\circ}$, Doutorando Programa de Pós-graduação em Produção Vegetal, Universidade Federal do Paraná.

${ }^{3}$ Eng $^{\circ}$-Agr ${ }^{\circ}$. Professor, Doutor, Departamento de Fitotecnia e Fitossanitarismo, Universidade Federal do Paraná. Rua dos Funcionários, 1540 CEP 80035-050, Curitiba-PR, Brasil.
} 
SPADER, V. et al. Fluxos de emergência de azevém em...

\section{INTRODUÇÃO}

O sistema de produção Integração Lavoura -Pecuária é utilizado por muitos produtores rurais na região Centro-Sul do estado do Paraná, como uma alternativa economicamente viável, de diversificação do negócio dentro das propriedades. Neste sistema, as áreas são divididas de forma que, partes são ocupadas com culturas para produção de grãos e parte com culturas forrageiras para alimentação de animais, principalmente bovinos. Entre as culturas para produção de grãos estão: milho e soja no verão e trigo e cevada no inverno.

$\mathrm{O}$ azevém (Lollium multiforum Lam.) é muito importante como forrageira, devido as suas características nutricionais, produtivas e de adaptação à pressão de pastejo (Brede \& Duich, 1984; Callow et al., 2003). Além disso, esta espécie apresenta sementes com dormência (Lodge, 2004; Venuto et al., 2002), o que facilita a ressemeadura natural nos anos seguintes, reduzindo os custos da pastagem (Chambers \& Macmohon, 1994).

Entretanto, neste sistema, durante o inverno, a rotação mais utilizada na região inclui dois anos consecutivos com pastagem e outros dois anos com culturas de grãos (trigo e cevada). Assim, nos anos em que se cultiva trigo e cevada, o azevém germina juntamente com estas culturas e exerce forte interferência sobre as mesmas, caso não seja controlado no momento correto.

A época de semeadura da cevada e do trigo, na região de Guarapuava, é durante os meses de junho e julho, dependendo da cultura e da cultivar. Este planejamento leva em consideração, principalmente, o ciclo vegetativo das cultivares, de modo que as mesmas iniciem a fase reprodutiva após o dia 15 de setembro, período em que a probabilidade de ocorrência de geadas é baixa. Isso é importante porque durante a fase reprodutiva, tanto a cevada quanto o trigo, são bastante sensíveis a geadas, sendo que, dependendo da intensidade, a geada pode causar perdas de até $100 \%$ destas culturas.

A identificação do comportamento da emergência do azevém, oriunda do banco de sementes do solo, durante o outono e o inverno, facilita o manejo desta espécie (Chambers \& Macmahon, 1994; Lodge, 2004) diminui sua interferência nas culturas de trigo e cevada (Hoskins et al., 2005) e reduz os custos com herbicidas, principalmente com os pós-emergentes seletivos às culturas (Dear et al., 2006). Deste modo, conforme a disponibilidade de cultivares de trigo e de cevada para semeadura em várias épocas é possível aguardar a ocorrência dos maiores fluxos de emergência do azevém, fazer a dessecação e só depois semear as culturas (Bean et al., 2005), reduzindo o seu potencial de interferência sobre o trigo e a cevada. Considerando o exposto, o objetivo deste trabalho foi determinar os fluxos de emergência do azevém, em área de integração lavoura pecuária na região de Guarapuava- PR.

\section{MATERIAL E MÉTODOS}

O estudo foi desenvolvido em área da Fun- dação Agrária de Pesquisa Agropecuária - FAPA, localizada no município de Guarapuava-PR, latitude $25^{\circ} 23^{\prime} 36^{\prime \prime} \mathrm{S}$, longitude $51^{\circ} 27^{\prime} 19^{\prime \prime} \mathrm{W}$ e altitude aproximada de $1100 \mathrm{~m}$. O solo do local é característico da região sendo classificado como Latossolo Bruno Distroférrico (EMBRAPA, 2006). O clima regional é classificado como Cfb - subtropical mesotérmico úmido, de acordo com a classificação de Köeppen. Os dados referentes à temperatura média do ar, temperatura do solo e precipitação foram obtidos por meio de uma estação meteorológica instalada próxima ao local do experimento.

O delineamento experimental foi o de blocos ao acaso, com seis repetições. Os tratamentos foram constituídos por 9 épocas de quantificação da emergência do azevém, a cada 15 dias, iniciando no dia 15 de abril e finalizando no dia 15 de setembro.

As quantificações dos fluxos de emergência foram realizadas por meio de lançamento de um quadrado de ferro com $0,5 \mathrm{~m}^{2}(0,71 \times 0,71 \mathrm{~m})$, aleatoriamente dentro de cada unidade experimental, contando-se as plantas emergidas na área do quadrado. O tamanho da parcela foi de $10 \times 10 \mathrm{~m}$. Após cada avaliação dos fluxos de emergência, toda a área experimental foi tratada com o herbicida paraquat $200 \mathrm{~g}$ i.a. (1,1'-dimetil-4,4'-bipiridilio dicloreto) (Gramoxone 200 - Syngenta Proteção de Cultivos Ltda.) na dosagem de até $0,5 \mathrm{~L} \mathrm{ha}^{-1}$, para eliminar todas as plantas presentes na área.

No final do experimento, os dados de fluxo de emergência foram analisados pelo método proposto por Blanco et al. (1994). Neste caso, atribuiuse o valor de 100 ao somatório total de indivíduos observados durante todo o período de avaliação e calculou-se o percentual observado em cada época de avaliação em relação a este valor, permitindo assim, a construção de um modelo de emergência da espécie.

\section{RESULTADOS E DISCUSSÃO}

A partir da avaliação dos dados climáticos da região de Guarapuava (PR) determinou-se a média mensal da precipitação pluvial ao longo do período de condução do experimento (Figura 1), ou seja, de meados de maio até meados de setembro de 2011. Os resultados demonstram que os meses que correspondem ao outono apresentam índices baixos de pluviosidade, destacando-se o mês de maio com $27 \mathrm{~mm}$, estando abaixo da média histórica para a região, a qual segundo Wagner (2009), (avaliando dados pluviométricos da região referentes ao período de 1984 a 2008) os meses de maio apresentam índices intermediários em torno de $170 \mathrm{~mm}$.

As condições climáticas do inverno para a região de Guarapuava apresentam os menores valores de precipitação que são sempre inferiores a $130 \mathrm{~mm}$, sendo que o mês de agosto apresenta índice de $88 \mathrm{~mm}$ que é a menor média anual (Wagner, 2009). Porém, o ano de 2011 apresentou índices superiores de precipitação para o inverno, destacando-se o mês de agosto, o qual apresentou média de 194,5 mm, estando bem acima da média histórica para a região. 


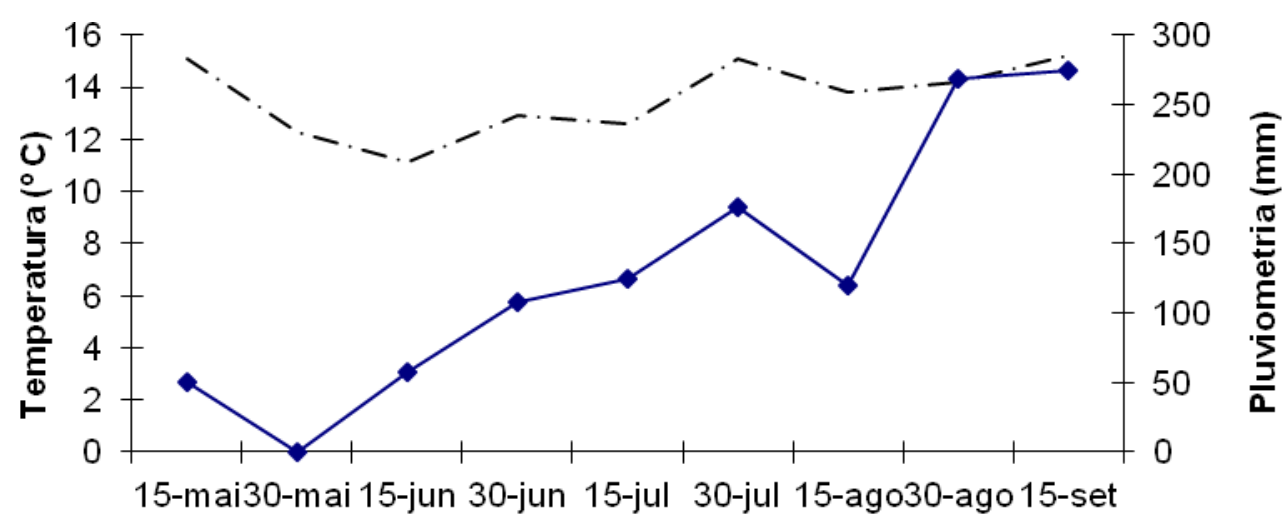

Datas de avaliação

$-\cdot-$ Temperatura média de 15 dias $\left({ }^{\circ} \mathrm{C}\right) \multimap$ Pluviometria acumulada em 15 dias $(\mathrm{mm})$

FIGURA 1. Temperatura média $\left({ }^{\circ} \mathrm{C}\right)$ no período, pluviometria acumulada $(\mathrm{mm})$ nas diferentes datas de avaliação do fluxo de emergência do azevém (L. multiflorum Lam.) em sistema de integração lavoura-pecuária. Guarapuava-PR, 2011.

As médias de temperatura encontraram-se dentro dos valores médios esperados para a região, sendo o mês de agosto com temperatura média de $14^{\circ} \mathrm{C}$, foi o que se apresentou como a mais elevada até o início de setembro, quando ocorreu a temperatura média de $15,2^{\circ} \mathrm{C}$ (Figura 1 ).

O comportamento médio da temperatura na região entre o período de abril a setembro de 1984 a 2009 é inferior a $16^{\circ} \mathrm{C}$, sendo o mês de julho o mais frio, com temperatura média inferior a $13^{\circ} \mathrm{C}$ (Wagner, 2009). Estes dados são acompanhados pela chegada de energia solar, cuja a radiação solar apresenta menores índices de radiação no outono-inverno, sendo normais para a respectiva época do ano (Caramori, 2003). De acordo com os dados meteorológicos observados, ficou constatado que este ano foi atípico, principalmente referindo-se à precipitação, a qual foi superior à média histórica da região.

Com a redução do fluxo inicial, observouse significativa redução dos fluxos posteriores de emergência de plântulas de azevém, o que pode ser observado nas subsequentes contagens. Quando as sementes de espécies anuais de inverno, como o azevém, estão em dormência primária, logo após a dispersão, a germinação está restrita a estreitas amplitudes de baixas temperaturas (Akinola et al., 1998).

Na data da primeira avaliação para contagem do fluxo de emergência do azevém, verificouse um maior fluxo de emergência, apresentando cerca de $65 \%$ de plântulas, sendo que a partir da aplicação do herbicida paraquat observou-se uma diminuição de plântulas emergidas na área ao longo do tempo (Tabela 1).

$\mathrm{Na}$ segunda avaliação a porcentagem de plântulas emergidas já se encontrava em torno de $25 \%$ (Tabela 1). Desta forma, é possível afirmar que o clima pode influenciar no fluxo de emergência das sementes, entretanto esta influencia não é determinante (Lodge, 2004), pois a emergência das sementes nos ciclos posteriores está relacionada com as sementes ainda aptas a germinar que sobraram na área após as aplicações do herbicida.

TABELA 1. População média de plântulas $\left(\mathrm{m}^{2}\right)$, população acumulada de plântulas $\left(\mathrm{m}^{2}\right)$ e porcentagem de emergência de azevém ( $L$. multiflorum Lam.) em sistema de integração lavoura-pecuária. Guarapuava-PR, 2011.

\begin{tabular}{cccccccccc}
\hline \multirow{2}{*}{ Parâmetros } & \multicolumn{10}{c}{ Datas das avaliações } \\
\cline { 2 - 10 } & $15 /$ mai & $30 /$ mai & $15 /$ jun & $30 /$ jun & $15 /$ jul & $30 /$ jul & $15 /$ ago & $30 /$ ago & $15 /$ set \\
\hline População/m & 1340 & 492 & 60 & 60 & 24 & 40 & 24 & 48 & 12 \\
População acumulada/m $\mathrm{m}^{-2}$ & 1340 & 1832 & 1892 & 1952 & 1976 & 2016 & 2040 & 2088 & 2100 \\
Emergência (\%) & 64 & 24 & 2.9 & 2,9 & 1,15 & 1,9 & 1,15 & 2,3 & 0,6 \\
\hline
\end{tabular}


SPADER, V. et al. Fluxos de emergência de azevém em...

\section{REFERÊNCIAS}

1.AKINOLA, M. O., THOMPSON, K.; BUCKLAND, S. M. Soil Seed Bank of an Upland Calcareous Grassland After 6 Years of Climate and Management Manipulations. The Journal of Applied Ecology, n.35, p.544-552. 1998.

2.BEAM, J. B.; BARKER, W. L.; ASKEW, S. D. Italian ryegrass (Lolium multiflorum) control in newly seeded tall fescue. Weed Technology, n.19, p. 416-421. 2005.

3.BLANCO, H. G.; AREVALO, R. A.; BLANCO, F. M. G. Distribuição mensal da emergência de seis ervas daninhas em solos com e sem cultivos. Planta Daninha, v. 12, n. 2, p. 78-83. 1994.

4.BREDE, A. D.; DUICH, J. M. Initial mowing of Kentucky bluegrass - perennial ryegrass seedling turf mixtures. Agronomy Journal, n.6, p. 711-714. 1984.

5.CALLOW, M. N.; LOWE, K. F.; BOWDLER, T. M.; LOWE, S. A.; GOBIUS, N. R. Dry matter yield, forage quality and persistence of tall fescue (Festuca arundinacea) cultivars compared with perennial ryegrass (Lolium perenne) in a subtropical environment. Australian Journal of Experimental Agriculture, n. 43, p. 1093-1099. 2003.

6.CARAMORI, P. H. Zoneamento agrícola no Estado do Paraná. Corporativo: Instituto Agronômico do Paraná, Londrina: PR, 76p. 2003.

7.CHAMBERS, J. C.; MACMAHON, J. A. A Day in the Life of a Seed: Movements and Fates of Seeds and Their Implications for Natural and Managed Systems. Annual Review of Ecology and Systematics, n. 25, p. 263-292. 1994.

8.DEAR, B. S.; SANDRAL, G. A.; WILSON, B. C. D. Tolerance of perennial pasture grass seedlings to pre- and postemergent grass herbicides. Australian Journal of Experimental Agriculture, n. 46, p. 637-644. 2006.

9.EMBRAPA. Empresa Brasileira de Pesquisa Agropecuária. Centro Nacional de Pesquisa de Solos. Sistema brasileiro de classificação de solos. 2. ed. Rio de Janeiro. Embrapa Solos, 306 p. 2006.

10.HOSKINS, A. J.; YOUNG, B. G.; KRAUSZ, R. F.; RUSSIN, J. S. Control of Italian ryegrass (Lolium multiflorum) in winter wheat. Weed Technology, n. 19, p. 251-265. 2005.

11.LODGE, G. M. Seed dormancy, germination, seedling emergence, and survival of some temperate perennial pasture grasses in northern New South Wales. Australian Journal of Agricultural Research, n. 55, p. 345-355. 2004

12.OLIVEIRA, J. C. P.; MORAES, C. O. C. Cadeia forrageira para a região de campanha. In: Cadeias forrageiras regionais.

Porto Alegre: Caramuru, 203p. 1995.

13.VENUTO, B. C.; REDFEARN, D. D.; PITMAN, W. D.; ALISON, M. W. Seed variation among annual ryegrass cultivars in south-eastern USA and the relationship with seedling vigour and forage production. Grass and Forage Science, n. 57, v. 4, p. 305-311. 2002.

14.WAGNER, M. V. Características hidroclimáticas para a cultura do milho em Guarapuava-PR. Dissertação de Mestrado. Universidade Estadual do Centro-Oeste. 2009. 